\title{
ON A TOPOLOGICAL CHARACTERIZATION OF PRÜFER $v$-MULTIPLICATION DOMAINS AMONG ESSENTIAL DOMAINS
}

\author{
CARMELO ANTONIO FINOCCHIARO AND FRANCESCA TARTARONE
}

\begin{abstract}
In this paper, we characterize the Prüfer $v$-multiplication domain as a class of essential domains verifying an additional property on the closure of some families of prime ideals, with respect to the constructible topology.
\end{abstract}

Introduction. The notion of the Prüfer domain, introduced by H. Prüfer in 1932, plays a central role in the theory of integrally closed domains. In fact, it globalizes the concept of the valuation domain in the sense that a domain is Prüfer if and only if it is locally a valuation domain (i.e., all its localizations at prime ideals are valuation domains). There is vast literature about the investigation of the multiplicative structure of ideals in Prüfer domains (for deeper insights on recent developments on this topic, see $[\mathbf{1 0}, \mathbf{2 4}])$. The notion of the Prüfer v-multiplication domain (abbreviated $\mathrm{P} v \mathrm{MD}$ ) was introduced to enlarge the class of Prüfer domains (for instance, two-dimensional regular domains are $\mathrm{P} v \mathrm{MD}$ but not Prüfer). More precisely, an integral domain is a $\mathrm{P} v \mathrm{MD}$ if and only if it is $t$-locally a valuation domain, i.e., each localization at $t$-prime ideals is a valuation domain (Section 1). Here, we just point out that the condition of being $t$-locally a valuation domain is certainly weaker than being locally a valuation domain because it involves a subset of the prime spectrum of a domain. Other interesting examples of $\mathrm{P} v \mathrm{MD}$ 's, besides Prüfer domains, are, for instance, $\mathbb{Z}[X]$ and, more generally, Krull domains.

Griffin [15] gives a very simple characterization of the P $v$ MDs with the $t$-finite character, i.e., each nonzero element of $D$ is contained

2010 AMS Mathematics subject classification. Primary 13A15, 13A18, 13F05, $54 \mathrm{~A} 20$.

Keywords and phrases. Prüfer domain, constructible topology, essential domain, ring of integer-valued polynomials.

Received by the editors on October 14, 2014.

DOI:10.1216/JCA-2016-8-4-513 Copyright (C)2016 Rocky Mountain Mathematics Consortium 
in finitely many $t$-maximal ideals. In this case, they are exactly the essential domains with the $t$-finite character (Theorem 2.2).

But the essential property for a domain $D$ is not, in general, equivalent to saying that $D$ is a $\mathrm{P} v \mathrm{MD}$. An important example of this fact is given by Heinzer and Ohm [17]. We have gone through this construction in order to understand what is missing in this essential domain for it not to be a PvMD. Then we have used this observation to give a general characterization of $\mathrm{P} v \mathrm{MDs}$ among the essential domains.

The central result of this paper is Theorem 2.4 in which we specifically describe $\mathrm{P} v \mathrm{MDs}$ as a subclass of essential domains that verifies an additional condition regarding ultrafilter limits of suitable families of prime ideals.

This theorem is, on the one hand, a generalization to any essential domain of the above-mentioned result by Griffin on domains with $t$-finite character and, on the other hand, it gives a topological explanation of what goes wrong with the Heinzer-Ohm example of an essential domain that is not a $\mathrm{P} v \mathrm{MD}$.

In Corollary 2.11, we compare the $\mathrm{P} v \mathrm{MD}$ property among domains with different quotient fields. In particular, we give a result in the case in which these quotient fields $K$ and $L$ form an algebraic extension $K \subseteq L$.

An interesting yet still open question is when a family of P $v$ MDs $\left\{D_{i}: i \in I\right\}$ is such that the intersection

$$
D=\bigcap_{i \in I} D_{i}
$$

is a $\mathrm{P} v \mathrm{MD}$. This does not happen even in very simple cases like the intersection of two P $v$ MD's (for instance, domains of the type $V \cap \mathbb{Q}[X]$, where $V$ is a valuation overring of $\mathbb{Z}[X]$, are quite often non-P $v \mathrm{MD})$.

In Theorem 2.14 and Corollary 2.15 we partially answer this question. In particular, we show that, if the family is finite and $D$ is "essential with respect to each $D_{i}$," then $D$ is a $\mathrm{P} v \mathrm{MD}$.

An interesting application of Theorem 2.4 is given in Section 3 with regard to the ring of integer-valued polynomials over a domain $D$,

$$
\operatorname{Int}(D)=\{f \in K[X]: f(D) \subseteq D\} .
$$


Both problems of characterizing when $\operatorname{Int}(D)$ is Prüfer or $\mathrm{P} v \mathrm{MD}$ have been investigated in the last 20 years (see, for instance, $[\mathbf{3}, \mathbf{4}, \mathbf{2 1}]$ ).

Here, we discuss the $\mathrm{P} v \mathrm{MD}$ property of $\operatorname{Int}(D)$. In Theorem 3.7, we refine the general characterization of a domain $D$ such that $\operatorname{Int}(D)$ is a $\mathrm{P} v \mathrm{MD}$ given in [3]. More precisely, we show that one of the three equivalent conditions of [3, Theorem 3.4] posed on $D$ can be deleted by putting an extra hypothesis on the localizations $\operatorname{Int}\left(D_{P}\right)$ (for $P \in t$-Spec $(D)$ ).

Most of the results presented in this paper are topological in nature, and their proofs are often based on techniques involving the constructible topology. For relevant contributions on this circle of ideas see, for instance, [7, 25].

1. Preliminaries. With the term ring, we will always mean a commutative ring with identity and, as usual, we denote by $\operatorname{Spec}(A)$ the set of all prime ideals of a ring $A$. For any ring homomorphism $f: A \rightarrow B$, we shall denote by

$$
f^{\star}: \operatorname{Spec}(B) \longrightarrow \operatorname{Spec}(A)
$$

the canonical map, induced by $f$.

Ultrafilter Limit Points. Given a set $X$, we recall that an ultrafilter on $X$ is a collection $\mathscr{U}$ of subsets of $X$ such that:

(1) $\emptyset \notin \mathscr{U}$.

(2) If $Y, Z \in \mathscr{U}$, then $Y \cap Z \in \mathscr{U}$.

(3) If $Y \in \mathscr{U}$ and $Y \subseteq Z \subseteq X$, then $Z \in \mathscr{U}$.

(4) For any $Y \subseteq X$, either $Y \in \mathscr{U}$ or $X-Y \in \mathscr{U}$.

We remind the reader in the following remark about some basic properties of ultrafilters that will be useful.

Remark 1.1. Let $X$ be a set.

(i) If $\mathscr{F}$ is a collection of sets with the finite intersection property, then $\mathscr{F}$ extends to some ultrafilter $\mathscr{U}$ on $X$, i.e., $\mathscr{U} \supseteq \mathscr{F}$.

(ii) If $x \in X$, the collection of sets $\mathscr{U}_{x}:=\{Y \subseteq X: x \in Y\}$ is an ultrafilter on $x$, called a principal ultrafilter. From the definitions, 
it easily follows that an ultrafilter is principal if and only if it contains a finite set.

(iii) An ultrafilter $\mathscr{U}$ on $X$ is non principal if $\mathscr{U} \neq \mathscr{U}_{x}$, for any $x \in X$. A straightforward application of Zorn's lemma shows that $X$ admits non principal ultrafilters if and only if it is infinite.

Now, let $A$ be a ring. Unless otherwise specified, we endow $\operatorname{Spec}(A)$ with the Zariski topology, whose closed sets are of the form

$$
V(\mathfrak{a}):=\{\mathfrak{p} \in \operatorname{Spec}(A): \mathfrak{a} \subseteq \mathfrak{p}\},
$$

for any ideal $\mathfrak{a}$ of $A$. For any $Y \subseteq \operatorname{Spec}(A)$, we shall denote by $\mathrm{Cl}^{\mathrm{c}}(Y)$ the closure of $Y$, with respect to the constructible topology, that is, the smallest topology for which any set of the form

$$
D(f):=\{\mathfrak{p} \in \operatorname{Spec}(A): f \notin \mathfrak{p}\}, \quad f \in A,
$$

is clopen. It follows easily by definition that a basis of clopen sets for the constructible topology is:

$$
\{D(f) \cap V(\mathfrak{a}): f \in A, \mathfrak{a} \text { finitely generated ideal of } A\}
$$

Recently, a relation between the constructible topology and the notion of an ultrafilter limit point has been shown independently [6, 11]. More precisely, let $Y$ be a nonempty subset of $\operatorname{Spec}(A)$, and let $\mathscr{U}$ be an ultrafilter on $Y$. By [3, Lemma 2.4], the set

$$
Y_{\mathscr{U}}:=\{a \in A: V(a) \cap Y \in \mathscr{U}\}
$$

is a prime ideal of $A$, called the ultrafilter limit point of $Y$, with respect to $\mathscr{U}$. By [6, Corollary 2.16] and [11, Theorem 8], a set is closed with respect to the constructible topology if and only if it contains all of its ultrafilter limit points. Moreover, by [6, Proposition 2.12], we have

$$
\mathrm{Cl}^{\mathrm{c}}(Y)=\left\{Y_{\mathscr{U}}: \mathscr{U} \text { ultrafilter on } Y\right\}
$$

for every subset $Y$ of $\operatorname{Spec}(A)$.

The $t$-operation. Given an integral domain $D$ with a quotient field $K$ we have the following standard terminology. For each nonzero (fractional) ideal $I$ of $D$ the divisorial closure of $I$ is the ideal $I^{v}=$ $(D:(D: I))$, where

$$
(D: I):=\{x \in K: x I \subseteq D\} .
$$


The $t$-closure of $I$ is

$I^{t}=\bigcup\left\{J^{v}: J\right.$ is a finitely generated nonzero ideal and $\left.J \subseteq I\right\}$.

The ideal $I$ is called a $t$-ideal if either $I=(0)$ or $I=I^{t}$, and it is a $t$-prime if it is prime and a $t$-ideal (usually the notion of a $t$-ideal is given for nonzero fractional ideals, but here it will be convenient to declare (0) a $t$-ideal, by definition). A $t$-maximal ideal is a $t$-ideal which is maximal among the proper $t$-ideals of $D$. A $t$-maximal ideal is $t$-prime, and a proper $t$-ideal is always contained in a $t$-maximal ideal. We denote by $t$-Max $(D)$ the set of the $t$-maximal ideals of $D$ and by $t$-Spec $(D)$ the set of $t$-prime ideals of $D$. For background material on $t$-operation see, for instance, $[\mathbf{1 4}, \mathbf{1 9}]$.

2. Main results. Let $D$ be an integral domain. A valuation overring of $D$ is said to be essential for $D$ if it is a localization of $D$. A prime ideal of $D$ is essential if it is the center of an essential valuation overring of $D$. A collection $\mathcal{V}$ of overrings of $D$ is said to be an essential representation of $D$ if

$$
D=\bigcap\{V: V \in \mathcal{V}\}
$$

and each member of $\mathcal{V}$ is essential for $D$. Recall that $D$ is said to be essential if it has an essential representation. Denote by $\mathcal{E}(D)$ the essential prime spectrum of $D$, i.e.,

$$
\mathcal{E}(D):=\left\{\mathfrak{p} \in \operatorname{Spec}(D): D_{\mathfrak{p}} \text { is a valuation domain }\right\}
$$

Remark 2.1. We recall the following well-known facts.

(i) Any domain $D$ can be represented as

$$
D=\bigcap_{M \in t-\operatorname{Max}(D)} D_{M}
$$

[15, Proposition 4].

(ii) An integrally closed domain is a Prüfer domain if and only if every ideal is a $t$-ideal [14, Proposition 34.12]. In particular, every ideal of a valuation domain is a $t$-ideal.

(iii) A $\mathrm{P} v \mathrm{MD}$ is always essential because $D_{M}$ is a valuation domain for each $M \in t$-Max $(D)([\mathbf{2 0}$, Theorem 3.2]). 
(iv) For any integral domain $D$ the following inclusion $\mathcal{E}(D) \subseteq$ $t$-Spec $(D)$ holds, by [20, Lemma 3.17].

(v) There exist essential domains that are not PvMD. An example is given by Heinzer and Ohm ([17], see also the following Example 2.3).

(vi) A domain $D$ has the $t$-finite (respectively, finite) character if each nonzero element $x \in D$ belongs to finitely many $t$-maximal (respectively, maximal) ideals of $D$.

$\mathrm{P} v \mathrm{MD}$ 's may not have the $t$-finite character; for instance, take $\mathbb{Z}+X \mathbb{Q}[X]$, which is a Bezout domain, hence a $\mathrm{P} v \mathrm{MD}$.

(vii) By [3, Lemma 2.4, Proposition 2.5] or [12, Corollary 2.10], every nonzero ultrafilter limit of a family of $t$-prime ideals is a $t$-prime ideal. Since we have set $(0)$ to be a $t$-ideal, we have that every ultrafilter limit of a family of $t$-prime ideals is a $t$-prime ideal, that is, $t$ - $\operatorname{Spec}(D)$ is closed, with respect to the constructible topology [11, Theorem 8].

Thus, if $D$ is a $\mathrm{P} v \mathrm{MD}$, then $\mathcal{E}(D)$ is closed with respect to the constructible topology, since $\mathcal{E}(D)=t$-Spec $(D)$.

In Theorem 2.4, we characterize P $v$ MDs in terms of the closure (with respect to the constructible topology) of a suitable subset of $\mathcal{E}(D)$.

We say that a collection of overrings $\mathcal{O}$ of $D$ is locally finite if, for any nonzero element $x \in D$, the set $\{B \in \mathcal{O}: x$ is not invertible in $B\}$ is finite. Recall that an integral domain is a Krull-type domain if it is an essential domain and it has a locally finite essential representation. The following result characterizes a Krull-type domain.

Theorem 2.2 ([15, Proposition 4, Theorems 5 and 7]). Let $D$ be an integral domain. Then, the following conditions are equivalent.

(i) D is a Krull-type domain.

(ii) $D$ is a $\mathrm{P} v \mathrm{MD}$ with $t$-finite character.

An example of a non $\mathrm{P} v \mathrm{MD}$ essential domain is given in [17]. Using this example, we will be able to outline the topological aspects that will be central in Theorem 2.4. 
Example 2.3 ([17]). Let $K$ be a field, and let $X_{0}, X_{1} \ldots, X_{n}, \ldots, T, U$ be an infinite and countable collection of intedeterminates over $K$. Set $\mathcal{X}:=\left\{X_{n}: n \in \mathbb{N}\right\}$, and consider the Krull domain $R:=$ $K(\mathcal{X})[T, U]_{(T, U)}$. Moreover, for any $i \in \mathbb{N}$, let $v_{i}$ be the valuation on $L:=K(\mathcal{X}, T, U)$ such that

$$
v_{i}\left(K\left(\left\{X_{j}: j \neq i\right\}\right)\right)=\{0\}
$$

and

$$
v_{i}\left(X_{i}\right)=v_{i}(T)=v_{i}(U):=1
$$

(define $v_{i}$ on polynomials in the canonical way, i.e., just by taking the infimum of the value of each monomial and extend it to $L$ ). For any $i \in \mathbb{N}$, let $V_{i}$ be the DVR associated to $v_{i}$, let $\mathfrak{m}_{i}$ be its maximal ideal, and set

$$
D:=R \cap \bigcap_{i \in \mathbb{N}} V_{i} .
$$

In [17], the authors show that $D$ is an essential domain that is not a $\mathrm{P} v \mathrm{MD}$. More precisely, they show that

$$
Y:=\{\mathfrak{p} \cap D: \mathfrak{p} \text { height-one prime of } R\} \cup\left\{\mathfrak{m}_{i} \cap D: i \in \mathbb{N}\right\}
$$

is a collection of essential prime ideals of $D$.

We will now give a new proof of the fact that $D$ is not a P $v \mathrm{MD}$, and it will help to understand the characterization given in Theorem 2.4. As a matter of fact, set

$$
\mathscr{F}:=\left\{V(f) \cap Y: f \in D \cap(T, U) K(\mathcal{X})[T, U]_{(T, U)}\right\},
$$

and take finitely many elements $f_{1}, \ldots, f_{h} \in D \cap(T, U) K(\mathcal{X})[T, U]_{(T, U)}$. Then there is a natural integer $n$ such that

$$
\left(f_{1}, \ldots, f_{n}\right) D \subseteq D \cap(T, U) K\left(X_{0}, \ldots, X_{n}\right)[T, U]_{(T, U)} .
$$

Furthermore, it is straightforward to show that the inclusion

$$
D \cap(T, U) K\left(X_{0}, \ldots, X_{n}\right)[T, U]_{(T, U)} \subseteq \mathfrak{m}_{i} \cap D
$$

holds for any $i>n$. It follows that

$$
\mathfrak{m}_{i} \cap D \in V\left(\left(f_{1}, \ldots, f_{n}\right) D\right) \cap Y, \quad \text { for any } i>n,
$$


i.e., the collection of sets $\mathscr{F}$ has the finite intersection property. Then there is an ultrafilter $\mathscr{U}$ on $Y$ such that $\mathscr{F} \subseteq \mathscr{U}$. By definition, the ultrafilter limit point

$$
Y_{\mathscr{U}}:=\{f \in D: V(f) \cap Y \in \mathscr{U}\}
$$

satisfies the inclusion $D \cap(T, U) K(\mathcal{X})[T, U]_{(T, U)} \subseteq Y_{\mathscr{U}}$. It follows that $D_{Y_{\mathscr{U}}} \subseteq R$, and then $D_{Y_{\mathscr{U}}}$ is not a valuation domain. Moreover, keeping in mind the equality $(\diamond)$ and Remark 2.1, we have

$$
Y_{\mathscr{U}} \in \mathrm{Cl}^{\mathrm{c}}(Y) \subseteq \mathrm{Cl}^{\mathrm{c}}(t-\operatorname{Spec}(D))=t-\operatorname{Spec}(D) .
$$

Thus, $D$ is not a $\mathrm{P} v \mathrm{MD}$.

Observe that we have found the bad $t$-prime ideal $Y_{\mathscr{U}}$ that makes $D$ fail to be a $\mathrm{P} v \mathrm{MD}$ in the closure of the set of the centers of an essential representation of $D$.

In view of Theorem 2.2 and the previous example, the following question arises naturally. Let $D$ be an essential domain with an essential representation $\mathcal{V}$. Is it possible to put extra conditions on $\mathcal{V}$, weaker than locally finiteness, in order to get that $D$ is a $\mathrm{P} v \mathrm{MD}$ ?

Such conditions do exist in the literature; for instance, in [27, Lemma 8], the author proves that an essential domain $D$ is a $\mathrm{P} v \mathrm{MD}$ if and only if it is a $v$-finite conductor domain, i.e., for every pair $a, b \in D \backslash\{0\} a D \cap b D$ is a $v$-ideal of finite type.

In the following, we present a new condition, topological in nature, for characterizing $\mathrm{P} v \mathrm{MDs}$ among essential domains.

Theorem 2.4. Let $D$ be an integral domain and $\mathcal{E}(D)$ the essential prime spectrum of $D$. Then, the following conditions are equivalent.

(i) $D$ is $a \mathrm{P} v \mathrm{MD}$.

(ii) $D$ is an essential domain, and there is an essential representation $\mathcal{V}:=\left\{D_{\mathfrak{p}}: \mathfrak{p} \in Y\right\}$ of $D$, for some $Y \subseteq \operatorname{Spec}(D)$, such that $\mathrm{Cl}^{\mathrm{c}}(Y) \subseteq \mathcal{E}(D)$.

Proof. (i) $\Rightarrow$ (ii). Assume that $D$ is a $\mathrm{P} v \mathrm{MD}$, and take $Y:=$ $t$-Spec $(D)$. Then, $Y$ is closed with respect to the constructible topology by Remark 2.1 (vii) and, by assumption, $Y \subseteq \mathcal{E}(D)$. Finally, it suffices to note that $\left\{D_{\mathfrak{p}}: \mathfrak{p} \in Y\right\}$ is an essential representation of $D$. 
(ii) $\Rightarrow$ (i). Let $\mathfrak{m}$ be a $t$-maximal ideal of $D$, and set

$$
\mathscr{F}:=\{V(x) \cap Y: x \in \mathfrak{m}\}
$$

We claim that $\mathscr{F}$ has the finite intersection property. If not, there exist elements $x_{1}, \ldots, x_{n} \in \mathfrak{m}$ such that $V\left(x_{1}, \ldots, x_{n}\right) \cap Y=\emptyset$. Thus, if $\mathfrak{a}:=\left(x_{1}, \ldots, x_{n}\right) D$, for any $\mathfrak{p} \in Y$, there is an element $d \in \mathfrak{a}-\mathfrak{p}$. For any $x \in(D: \mathfrak{a})$ we have $d x \in D$, that is, $x \in D_{\mathfrak{p}}$. Since $\mathcal{V}$ is an essential representation, we infer that $(D: \mathfrak{a})=D$, i.e., $\mathfrak{a}^{v}=D$. Since $\mathfrak{a} \subseteq \mathfrak{m}$ and $\mathfrak{m} \in t$-Max $(D)$, we have $\mathfrak{a}^{v}=\mathfrak{m}^{t}=\mathfrak{m}=D$, a contradiction. Since $\mathscr{F}$ has the finite intersection property, we can pick an ultrafilter $\mathscr{U}$ on $Y$ extending $\mathscr{F}$. Then, it follows by definition $\mathfrak{m} \subseteq Y_{\mathscr{U}}:=\{x \in D: V(x) \cap Y \in \mathscr{U}\}$.

Now note that, by [20, Lemma 3.17(1)], we obtain the inclusion $Y \subseteq t$-Spec $(D)$. Moreover, as we have noted in Remark 2.1 (vii), $t$-Spec $(D)$ is closed, in the constructible topology, and thus, $\mathrm{Cl}^{\mathrm{c}}(Y) \subseteq$ $\mathrm{Cl}^{\mathrm{c}}(t-\operatorname{Spec}(D))=t-\operatorname{Spec}(D)$. By [6, Proposition 2.12], $Y_{\mathscr{U}} \in \mathrm{Cl}^{\mathrm{c}}(Y)$ and, a fortiori, $Y_{\mathscr{U}} \in t$-Spec $(D)$. Since $\mathfrak{m}$ is $t$-maximal, it follows that $\mathfrak{m}=Y_{\mathscr{U}}$. On the other hand, $\mathrm{Cl}^{\mathrm{c}}(Y) \subseteq \mathcal{E}(D)$, and thus, $D_{\mathfrak{m}}=D_{Y_{\mathscr{U}}}$ is a valuation domain. The proof is now complete.

Corollary 2.5. Let $D$ be an essential domain that admits an essential representation $\mathcal{V}$ such that the set of the centers in $D$ of the valuation domains in $\mathcal{V}$ is closed, with respect to the constructible topology. Then $D$ is a $\mathrm{P} v \mathrm{MD}$.

Proof. Apply Theorem 2.4.

Corollary 2.6. An integral domain $D$ is a $\mathrm{P} v \mathrm{MD}$ if and only if $D$ is essential and $\mathcal{E}(D)$ is closed, with respect to the constructible topology.

Proof. If $D$ is a $\mathrm{P} v \mathrm{MD}$, then $\mathcal{E}(D)=t$-Spec $(D)$, and it is closed with respect to the constructible topology. Conversely, if $D$ is essential, then $\left\{D_{\mathfrak{p}}: \mathfrak{p} \in \mathcal{E}(D)\right\}$ is clearly an essential representation of $D$. Since, by assumption, $\mathcal{E}(D)$ is closed, the conclusion follows by Corollary 2.5.

Corollary 2.7. Let $D$ be an integral domain. Then, the following conditions are equivalent.

(i) $D$ is a $\mathrm{P} v \mathrm{MD}$. 
(ii) $D$ is an essential domain, and it admits an essential representation $\left\{D_{\mathfrak{p}}: \mathfrak{p} \in Y\right\}$, where $Y \subseteq \operatorname{Spec}(D)$ is compact, with respect to the Zariski topology.

Proof.

(i) $\Rightarrow$ (ii) follows by taking $Y:=t-\operatorname{Spec}(D)$.

(ii) $\Rightarrow$ (i). For any topological space $X$ and any subset $S$ of $X$, the set,

$$
S^{\text {gen }}:=\{x \in X: s \in \mathrm{Cl}(\{x\}) \text {, for some } s \in S\},
$$

is called a closure under generizations (or generic closure) of $S$. The properties of $S^{\text {gen }}$ are particularly interesting when $X$ is a spectral space and $S$ is compact. In this case, by [7, Proposition 2.6], $S^{\text {gen }}$ is closed, with respect to the constructible topology.

Now, let $Y$ be a compact subspace of $\operatorname{Spec}(D)$ such that $\left\{D_{\mathfrak{p}}\right.$ : $\mathfrak{p} \in Y\}$ is an essential representation of $D$. By, definition, the closure under generizations of $Y$ is the set

$$
Y^{\text {gen }}=\{\mathfrak{q} \in \operatorname{Spec}(D): \mathfrak{q} \subseteq \mathfrak{p}, \text { for some } \mathfrak{p} \in Y\} .
$$

Of course, $\mathcal{V}:=\left\{D_{\mathfrak{q}}: \mathfrak{q} \in Y^{\mathrm{gen}}\right\}$ is still a representation of $D$ because $Y^{\text {gen }}$ contains $Y$. We claim that $\mathcal{V}$ is also essential since, if $\mathfrak{q} \in Y^{\text {gen }}$ and $\mathfrak{p} \in Y$ is such that $\mathfrak{q} \subseteq \mathfrak{p}$, then $D_{\mathfrak{p}}$ is a valuation domain and $D_{\mathfrak{q}} \supseteq D_{\mathfrak{p}}$. The conclusion follows by applying Theorem 2.4 and keeping in mind the above discussion.

We now give a natural application of Corollary 2.5.

Example 2.8 (see [9, Theorem 4.1]). Let $V$ be a valuation domain with residue field $k$, and let $\pi: V \rightarrow k$ be the canonical projection. Let $D$ be a $\mathrm{P} v \mathrm{MD}$ whose quotient field is $k$. Consider the following pullback diagram:

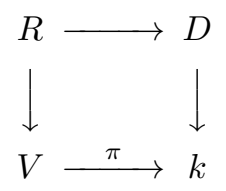

We claim that the ring $R:=\pi^{-1}(D)$ is a $\mathrm{P} v \mathrm{MD}$. 
By [9, Corollary 1.9], $\pi^{-1}(\mathfrak{p})$ is a $t$-prime ideal of $R$, for any $t$-prime ideal $\mathfrak{p}$ of $D$, and it is easy to check that $\pi^{-1}\left(D_{\mathfrak{p}}\right)=R_{\pi^{-1}(\mathfrak{p})}$.

Thus, keeping in mind that $D$ is a $\mathrm{P} v \mathrm{MD}$ whose quotient field is $k$, [8, Theorem $2.4(1)]$ implies that the collection

$$
\mathcal{V}:=\left\{R_{\pi^{-1}(\mathfrak{p})}: \mathfrak{p} \in t-\operatorname{Spec}(D)\right\}
$$

is an essential representation of $R$.

The centers in $R$ of the valuation domains of $\mathcal{V}$ are the inverse images $\pi^{-1}(\mathfrak{p})$, for $\mathfrak{p} \in t$-Spec $(D)$. This set is closed with respect to the constructible topology, by [1, Chapter 3, Exercise 29] and Remark 2.1 (vi). The conclusion follows by Corollary 2.5.

The following lemma will be useful for explaining the methodology of Griffin's Theorem 2.2 following from Theorem 2.4.

Lemma 2.9. Let $A$ be a ring and $Y$ an infinite subset of $\operatorname{Spec}(A)$ such that every nonzero element of $A$ belongs only to finitely many prime ideals of $Y$. Then, $A$ is an integral domain and $\mathrm{Cl}^{\mathrm{c}}(Y)=Y \cup\{(0)\}$.

Proof. Since $Y$ is infinite, take a non principal ultrafilter $\mathscr{U}$ on $Y$, and let

$$
Y_{\mathscr{U}}:=\{x \in A: V(x) \cap Y \in \mathscr{U}\}
$$

be the ultrafilter limit prime ideal of $Y$, with respect to $\mathscr{U}$ (see $[\mathbf{3}$, Lemma 2.4]). Thus, for any element $x \in Y_{\mathscr{U}}$, the set

$$
T:=V(x) \cap Y \in \mathscr{U} .
$$

Thus, $T$ is infinite since the ultrafilter is not principal and, since every nonzero element of $A$ only belongs to finitely many prime ideals of $Y$, it follows that $x=0$. This proves that $Y_{\mathscr{U}}=(0)$. Thus, $(0)$ is a prime ideal and so $A$ is an integral domain. Furthermore, $(0) \in \mathrm{Cl}^{\mathrm{C}}(Y)$. Since the equality $Y_{\mathscr{U}}=(0)$ holds for any non principal ultrafilter $\mathscr{U}$ on $Y$, the conclusion follows immediately from equality $(\diamond)$.

Remark 2.10. Now we observe that the nontrivial part (i) $\Rightarrow$ (ii) of Griffin's characterization of Krull-type domains (Theorem 2.2 (ii)) follows from Theorem 2.4. Suppose $D$ is a Krull-type domain, and let $\mathcal{V}:=\left\{D_{\mathfrak{p}}: \mathfrak{p} \in Y\right\}$ be an essential and locally finite representation of 
$D$ (for some subset $Y$ of $\operatorname{Spec}(D)$ ). Of course, for any $d \in D-\{0\}$, only finitely many prime ideals in $Y$ contain $d$. Thus, if $Y$ is infinite, by Lemma 2.9 , we have

$$
\begin{aligned}
& \mathrm{Cl}^{\mathrm{c}}(Y)=Y \cup\{0\} \subseteq \mathcal{E}(D) \\
&:=\left\{\mathfrak{p} \in \operatorname{Spec}(D): D_{\mathfrak{p}} \text { is a valuation domain }\right\} .
\end{aligned}
$$

If $Y$ is finite, it is clearly closed since the constructible topology is Hausdorff, in particular. Thus, in any case, we have $\mathrm{Cl}^{\mathrm{c}}(Y) \subseteq \mathcal{E}(D)$ and, by Theorem $2.4, D$ is a $\mathrm{P} v \mathrm{MD}$.

Note also that Theorem 2.2 (i) $\Rightarrow$ (ii) follows from the fact that an essential domain with a locally finite essential representation is a $v$ finite conductor domain.

Corollary 2.11. Let $K \subseteq L$ be an algebraic field extension, $A$ a $\mathrm{P} v \mathrm{MD}$ whose field of fractions is $K$ and $B$ an integrally closed essential domain with field of fractions L. Moreover, suppose that $B$ admits an essential representation $\mathcal{V}$ such that, for any $V \in \mathcal{V}$, the center of $V$ in $A$ is a $t$-ideal. Then $B$ is a $\mathrm{P} v \mathrm{MD}$.

Proof. Let $X$ be the subset of $\operatorname{Spec}(B)$ such that $\mathcal{V}=\left\{B_{\mathfrak{h}}: \mathfrak{h} \in X\right\}$, and let $\iota^{\star}: \operatorname{Spec}(B) \rightarrow \operatorname{Spec}(A)$ denote the map naturally induced by the inclusion $\iota: A \rightarrow B$. By [1, Chapter 3, Exercise 29], the map $\iota^{\star}$ is continuous (and closed) if $\operatorname{Spec}(A), \operatorname{Spec}(B)$ are equipped with the constructible topology, and then $\iota^{\star}\left(\mathrm{Cl}^{\mathrm{c}}(X)\right) \subseteq \mathrm{Cl}^{\mathrm{c}}\left(\iota^{\star}(X)\right)$. On the other hand, $\iota^{\star}(X)$ is clearly the set of all centers in $A$ of the valuation domains in $\mathcal{V}$, and thus, keeping in mind the assumption and applying [3, Lemma 2.4 and Proposition 2.5], we have $\mathrm{Cl}^{\mathrm{c}}\left(\iota^{\star}(X)\right) \subseteq t$-Spec $(A)$.

Now take a prime ideal $\mathfrak{p} \in \mathrm{Cl}^{\mathrm{c}}(X)$. Since we have $\iota^{\star}\left(\mathrm{Cl}^{\mathrm{c}}(X)\right) \subseteq$ $t$-Spec $(A), \mathfrak{p} \cap A$ is a $t$-prime ideal of $A$ and, since $A$ is a $\mathrm{P} v \mathrm{MD}, A_{\mathfrak{p} \cap A}$ is a valuation domain such that $A_{\mathfrak{p} \cap A} \subseteq B_{\mathfrak{p}}$. Then the integral closure ${\overline{A_{\mathfrak{p} \cap A}}}^{(L)}$ of $A_{\mathfrak{p} \cap A}$ in $L$ is a Prüfer domain whose field of fractions is $L$ and, since $B$ is integrally closed, we have ${\overline{A_{\mathfrak{p} \cap A}}}^{(L)} \subseteq B_{\mathfrak{p}}$. It follows that $B_{\mathfrak{p}}$ is a valuation domain, since it is a local overring of a Prüfer domain. Now it suffices to apply Theorem 2.4. 
Example 2.12. Let $\mathbb{Q} \subset K$ be a finite field extension, and consider a DVR overring $\left(V, M_{V}\right)$ of $\mathbb{Z}[X]$ such that $A=V \cap \mathbb{Q}[X]$ is a $\mathrm{P} v \mathrm{MD}$ [22, Theorem 5.8]. Let $\left(W, M_{W}\right)$ be an extension of $V$ to $K(X)$. Then $B=W \cap K[X]$ is a $\mathrm{P} v \mathrm{MD}$.

We must show that

$$
W \cap \bigcap_{Q \in \operatorname{Max}(K[X])} K[X]_{Q}
$$

is an essential representation of $B$. It is easy to check that $K[X]_{Q}=$ $B_{Q \cap B}$, for each $Q \in \operatorname{Max}(K[X])$ (these ideals $Q \cap B$ are exactly the uppers to the 0 of $B$ ).

Regarding $W$,

$$
M_{W} \cap B \supset M_{W} \cap A=M_{V} \cap A
$$

(since $W$ is an extension of $V$ ), and it is known that $A_{M_{V} \cap A}=V[\mathbf{2 2}$, Theorem 5.8 and Lemma 1.3 (2)]. Then $V \subset B_{M_{W} \cap B}$, and so $B_{M_{W} \cap B}$ is a valuation domain since it contains the integral closure of $V$ in $K$, that is, Prüfer [14, Theorem 22.3]. From dimension considerations, it follows that $B_{M_{W} \cap B}=W$.

Now $W$ is centered in $M_{V} \cap A$ that is a $t$-ideal of $A$ (since it is minimal over a principal ideal by [22, Proof of Lemma $1.3(1)])$. All the valuation overrings of $K[X]$ are centered in the upper to zero primes of $A$, which are also $t$-primes of $A$. Thus, $B$ is a $\mathrm{P} v \mathrm{MD}$ by Corollary 2.11.

Corollary 2.13 ([20, Corollary 3.9], [23, Proposition 5.1]). Let $A$ be $a \mathrm{P} v \mathrm{MD}$, and let $X$ be a nonempty collection of t-prime ideals of $A$. Then

$$
\bigcap\left\{A_{\mathfrak{p}}: \mathfrak{p} \in X\right\}
$$

is a $\mathrm{P} v \mathrm{MD}$.

Proof. Set

$$
B:=\bigcap\left\{A_{\mathfrak{p}}: \mathfrak{p} \in X\right\}
$$

and, for any prime ideal $\mathfrak{p} \in X$, set $\widetilde{\mathfrak{p}}:=\mathfrak{p} A_{\mathfrak{p}} \cap B$. Note that, since obviously $B_{\tilde{\mathfrak{p}}}=A_{\mathfrak{p}}$, for any $\mathfrak{p} \in X$, the collection of rings $\mathcal{V}:=\left\{B_{\widetilde{\mathfrak{p}}}: \mathfrak{p} \in X\right\}$ is an essential representation of $B$ such that $\widetilde{\mathfrak{p}} \cap A=\mathfrak{p}$ 
is a $t$-prime ideal of $A$. Then the statement follows immediately by Corollary 2.11 , just by taking $L:=K$.

Now we give a sufficient condition for an intersection of a family of $\mathrm{P} v \mathrm{MDs}$ to be a $\mathrm{P} v \mathrm{MD}$. Recall that a family $\mathcal{F}$ of subsets of a topological space $X$ is called a locally finite collection of sets if, for any $x \in X$, there is a neighborhood $U$ of $X$ such that

$$
\{F \in \mathcal{F}: F \cap U \neq \emptyset\}
$$

is finite.

Let $\left\{D_{i}: i \in I\right\}$ be a family of $\mathrm{P} v \mathrm{MDs}$, and set

$$
D:=\bigcap\left\{D_{i}: i \in I\right\} .
$$

We say that $D$ is essential, with respect to the family $\left\{D_{i}: i \in I\right\}$, if the canonical representation

$$
\left\{\left(D_{i}\right)_{\mathfrak{q}}: \mathfrak{q} \in t-\operatorname{Spec}\left(D_{i}\right), i \in I\right\}
$$

of $D$ is essential, i.e., $\left(D_{i}\right)_{\mathfrak{q}}$ is a valuation domain and a quotient ring of $D$. It follows immediately that, if $D$ is essential with respect to $\left\{D_{i}: i \in I\right\}$, then $D_{i}$ is an overring of $D$ for any $i \in I$.

Theorem 2.14. Let $\left\{D_{i}: i \in I\right\}$ be a nonempty collection of $\mathrm{P} v \mathrm{MDs}$, set

$$
D:=\bigcap\left\{D_{i}: i \in I\right\},
$$

and suppose that $D$ is essential with respect to the family $\left\{D_{i}: i \in I\right\}$. Assume also that, for any $\mathfrak{p} \in \operatorname{Spec}(D)$, there are an element $f \in D-\mathfrak{p}$ and a finitely generated ideal $\mathfrak{a} \subseteq \mathfrak{p}$ such that, only for finitely many indices $i \in I$, there may exist a $t$-prime ideal $\mathfrak{q}$ of $D_{i}$ such that $f \notin \mathfrak{q}$ and $\mathfrak{a} \subseteq \mathfrak{q} \cap D$. Then $D$ is a $\mathrm{P} v \mathrm{MD}$.

Proof. For any $i \in I$, let $\iota_{i}: D \rightarrow D_{i}$ denote the inclusion. Of course, the set of the centers of the canonical and essential representation

$$
\left\{\left(D_{i}\right)_{\mathfrak{q}}: \mathfrak{q} \in t-\operatorname{Spec}\left(D_{i}\right), i \in I\right\}
$$

of $D$ is

$$
X:=\left\{\mathfrak{q} \cap D: \mathfrak{q} \in t-\operatorname{Spec}\left(D_{i}\right), i \in I\right\}=\bigcup_{i \in I} \iota_{i}^{\star}\left(t-\operatorname{Spec}\left(D_{i}\right)\right) .
$$


Let $\mathfrak{p} \in \operatorname{Spec}(A)$. By assumption, the open neighborhood $D(f) \cap V(\mathfrak{a})$ (with respect to the constructible topology) intersects $\iota_{i}^{\star}\left(t-\operatorname{Spec}\left(D_{i}\right)\right)$ only for finitely many $i \in I$. Moreover, for any $i \in I$, the set $\iota_{i}^{\star}\left(t-\operatorname{Spec}\left(D_{i}\right)\right)$ is closed, with respect to the constructible topology, since $t$-Spec $\left(D_{i}\right)$ closed and $\iota_{i}^{\star}$ continuous. Thus,

$$
\left\{\iota_{i}^{\star}\left(t-\operatorname{Spec}\left(D_{i}\right)\right): i \in I\right\}
$$

is a locally finite family of closed sets of $\operatorname{Spec}(D)$. By [5, Theorem 1.1.11], we infer that $X$ is closed, with respect to the constructible topology. Thus, the conclusion follows immediately from Corollary 2.5.

The following results are immediate consequences of Theorem 2.14.

Corollary 2.15. Let $D_{1}, \ldots, D_{n}$ be $\mathrm{P} v \mathrm{MD} s$, set

$$
D:=D_{1} \cap \cdots \cap D_{n},
$$

and assume that $D$ is essential with respect to $\left\{D_{1}, \ldots, D_{n}\right\}$. Then $D$ is a $\mathrm{P} v \mathrm{MD}$.

Corollary 2.16. Let $\left\{D_{i}: i \in I\right\}$ be a nonempty family of $\mathrm{P} v \mathrm{MD}$, set

$$
D:=\bigcap\left\{D_{i}: i \in I\right\} \text {, }
$$

and suppose that $D$ is essential with respect to $\left\{D_{i}: i \in I\right\}$. Assume that at least one of the following properties is satisfied:

(i) For any $\mathfrak{p} \in \operatorname{Spec}(D)$, there is an element $f \in D-\mathfrak{p}$ such that, for only finitely many indices $i \in I$, there exists a t-prime ideal $\mathfrak{q}$ of $D_{i}$ such that $f \notin \mathfrak{q} \cap D$.

(ii) For any $\mathfrak{p} \in \operatorname{Spec}(D)$, there is a finitely generated ideal $\mathfrak{a}$ of $A$ contained in $\mathfrak{p}$ such that, for only finitely many indices $i \in I$, there exists a t-prime ideal $\mathfrak{q}$ of $D_{i}$ such that $\mathfrak{q} \cap D \supseteq \mathfrak{a}$.

Then $D$ is a $\mathrm{P} v \mathrm{MD}$.

The following example gives a direct application of Corollary 2.15.

Example 2.17. Let $\left(V, M_{V}\right)$ be a one-dimensional, discrete valuation overring of $\mathbb{Z}[X]$ such that $V \cap \mathbb{Q}[X]$ is $\mathrm{P} v \mathrm{MD}$, not Prüfer (see [22, 
Proposition 4.1 and Theorem 5.8]). Suppose that

$$
M_{V} \cap \mathbb{Z}[X]=(p, f(X)),
$$

where $p \in \mathbb{Z}$ is a prime integer and $f(X) \in \mathbb{Q}[X]$ is a non linear, monic and irreducible polynomial over $\mathbb{F}_{p}$ (the field with $p$ elements).

Then $A:=V \cap \operatorname{Int}(\mathbb{Z})$ is a $\mathrm{P} v \mathrm{MD}$, not Prüfer.

That $A$ is not Prüfer follows from the fact that its overring $V \cap \mathbb{Q}[X]$ is not Prüfer.

We recall that all the prime ideals of $\operatorname{Int}(\mathbb{Z})$ are either (0), uppers to 0 or maximals of the type

$$
\mathfrak{m}_{p, \alpha}=\left\{f \in \operatorname{Int}(\mathbb{Z}): f(\alpha) \in \widehat{p \mathbb{Z}_{(p)}}\right\},
$$

where $p \in \mathbb{Z}$ is prime and $\alpha \in \widehat{\mathbb{Z}_{(p)}}$ (the $p$-adic completion of $\mathbb{Z}$ ). It is also well known that

$$
\mathfrak{m}_{p, \alpha} \cap \mathbb{Z}[X]=(p, X-a),
$$

where $a \in \mathbb{Z}$ is such that $\alpha-a \in \widehat{p \mathbb{Z}_{(p)}}([2$, Remark V.2.6 (iiib)]). This implies that $\mathfrak{m}_{p, \alpha} \cap A \nsubseteq M_{V} \cap A$ since

$$
\mathfrak{m}_{p, \alpha} \cap \mathbb{Z}[X] \nsubseteq M_{V} \cap \mathbb{Z}[X]
$$

and $\mathbb{Z}[X] \subset A$.

The domain $\operatorname{Int}(\mathbb{Z})$ is Prüfer, so all its localizations at prime ideals are valuation domains.

Let us see that

$$
V \cap\left(\bigcap_{Q \in \operatorname{Spec}(\operatorname{Int}(\mathbb{Z}))} \operatorname{Int}(\mathbb{Z})_{Q}\right)
$$

is an essential representation of $A$.

If $Q \in \operatorname{Spec}(\operatorname{Int}(\mathbb{Z}))$ and $Q \cap \mathbb{Z}=(0)$, then

$$
\operatorname{Int}(\mathbb{Z})_{Q}=A_{Q \cap A}=\mathbb{Q}[X]_{(f)},
$$

where $f$ is such that

$$
Q=f \mathbb{Q}[X] \cap \operatorname{Int}(\mathbb{Z}) .
$$

Thus, $\operatorname{Int}(\mathbb{Z})_{Q}$ is a localization of $A$ that is a valuation domain. 
If $Q \cap \mathbb{Z}=(p)$ for some prime $p \in \mathbb{Z}$, then $Q=\mathfrak{m}_{p, \alpha}$, and there exists $\alpha \in \widehat{\mathbb{Z}_{(p)}}$. In this case,

$$
A_{\left(\mathfrak{m}_{p, \alpha} \cap A\right)}=V_{\left(A \backslash\left(\mathfrak{m}_{p, \alpha} \cap A\right)\right)} \cap \operatorname{Int}(\mathbb{Z})_{\left(A \backslash\left(\mathfrak{m}_{p, \alpha} \cap A\right)\right)} .
$$

But, since we have observed that $\mathfrak{m}_{p, \alpha} \cap A \nsubseteq M_{V}$, it follows that

$$
V_{\left(A \backslash\left(\mathfrak{m}_{p, \alpha} \cap A\right)\right)}=\mathbb{Q}(X),
$$

and so $A_{\left(\mathfrak{m}_{p, \alpha} \cap A\right)}=\operatorname{Int}(\mathbb{Z})_{\left(A \backslash\left(\mathfrak{m}_{p, \alpha} \cap A\right)\right)}$, that is a valuation domain since $\operatorname{Int}(\mathbb{Z})$ is Prüfer and $A_{\left(\mathfrak{m}_{p, \alpha} \cap A\right)}$ is a local overring of $\operatorname{Int}(\mathbb{Z})$.

Now, we will see that $V$ is a localization of $A$ at some prime ideal. Obviously, $V \nsupseteq \operatorname{Int}(\mathbb{Z})$, otherwise $V \cap \mathbb{Q}[X]$ would be Prüfer as an overring of $\operatorname{Int}(\mathbb{Z})$. We also have that $V$ is rational, i.e., its value group is contained in $\mathbb{Q}$. By [18, Lemma 1.3], we easily have that $A_{\mathfrak{M}_{V}}=V$, where $\mathfrak{M}_{V}$ is the center of $V$ in $A$.

By Corollary 2.5, we must show that the set of the centers in $A$ of

$$
\left\{\operatorname{Int}(\mathbb{Z})_{Q} ; Q \in \operatorname{Spec}(\operatorname{Int}(\mathbb{Z}))\right\} \cup\{V\}
$$

is closed with respect to the constructible topology, and this is equivalent to asking whether the set of the centers in $A$ of

$$
\left\{\operatorname{Int}(\mathbb{Z})_{Q} ; Q \in \operatorname{Spec}(\operatorname{Int}(\mathbb{Z}))\right\}
$$

is closed. Now, this set is exactly the image of $\operatorname{Int}(\mathbb{Z})$ under the map

$$
f^{\star}: \operatorname{Spec}(\operatorname{Int}(\mathbb{Z})) \longrightarrow \operatorname{Spec}(A), \quad P \longmapsto P \cap A,
$$

and so it is closed.

3. An application to integer-valued polynomials. Given a domain $D$ with quotient field $K$, the integer-valued polynomial ring on $D$ is the $\operatorname{ring} \operatorname{Int}(D):=\{f \in K[X]: f(D) \subseteq D\}$.

In [3] (for general domains) and [26] (for Krull-type domains) the authors study conditions on $D$ so that $\operatorname{Int}(D)$ is a $\mathrm{P} v \mathrm{MD}$.

Following the notation of [3], a $t$-prime ideal $P \in \operatorname{Spec}(D)$ is called int-prime if $\operatorname{Int}(D)_{(D \backslash P)} \neq D_{P}[X]$ (in the following, for simplicity of notation, we will set

$$
\operatorname{Int}(D)_{P}:=\operatorname{Int}(D)_{(D \backslash P)},
$$

for any prime ideal $P$ of $D)$. 
By Remark 2.1 (i), we have

$$
D=\bigcap_{P \in t-\operatorname{Spec}(D)} D_{P} .
$$

We define the following two subsets of $t-\operatorname{Spec}(D)$ as:

$$
\Lambda_{1}:=\left\{P \in t-\operatorname{Spec}(D): \operatorname{Int}(D)_{P}=D_{P}[X]\right\}
$$

and

$$
\Lambda_{0}:=\left\{P \in t-\operatorname{Spec}(D): \operatorname{Int}(D)_{P} \neq D_{P}[X]\right\}
$$

From [2, Proposition I.3.4], it follows that the ideals of $\Lambda_{0}$ are also maximal (since, by [3, Corollary 1.3], $|D / P|<\infty$ ).

We set

$$
D_{1}:=\bigcap_{P \in \Lambda_{1}} D_{P}
$$

and

$$
D_{0}:=\bigcap_{P \in \Lambda_{0}} D_{P} .
$$

From [3, Lemma 4.1], it follows that

$$
\operatorname{Int}(D)=D_{1}[X] \cap \operatorname{Int}\left(D_{0}\right) .
$$

If $\operatorname{Int}(D)$ is a $\mathrm{P} v \mathrm{MD}$, then $\operatorname{Int}\left(D_{0}\right)$ is Prüfer [3, Corollary 4.9], but this last condition is not sufficient to get that $\operatorname{Int}(D)$ is a $\mathrm{P} v \mathrm{MD}$, also assuming that $D$ is a $\mathrm{P} v \mathrm{MD}[\mathbf{3}$, Example 5.1].

If $D$ is Krull-type, the condition $\operatorname{Int}\left(D_{0}\right)$ is Prüfer is equivalent to asking whether $\operatorname{Int}(D)$ is a $\mathrm{P} v \mathrm{MD}$. This result is implicitly shown [26], but we give a more explicit proof of this fact in the next theorem.

Theorem 3.1. Let $D$ be a Krull-type domain. Then $\operatorname{Int}(D)$ is a $\mathrm{P} v \mathrm{MD}$ if and only if $\operatorname{Int}\left(D_{0}\right)$ is Prüfer.

Proof. If $\operatorname{Int}(D)$ is a $\mathrm{P} v \mathrm{MD}$, then we have already observed above that $\operatorname{Int}\left(D_{0}\right)$ is Prüfer. 
Suppose that $\operatorname{Int}\left(D_{0}\right)$ is Prüfer. Then $D_{0}$ is almost Dedekind by [2, Proposition VI.1.5]. If $P \in \Lambda_{0}$, then, by construction, $D_{P}$ is a local overring of $D_{0}$, and thus it is a DVR (since $D_{0}$ is almost Dedekind). Thus, $P$ is height one. From [26, Theorem 3.2], we know that, when $D$ is Krull-type, $\operatorname{Int}(D)$ is a $\mathrm{P} v \mathrm{MD}$ if and only if each $P \in \Lambda_{0}$ has height one. It follows that $\operatorname{Int}(D)$ is a $\mathrm{P} v \mathrm{MD}$.

Using Theorem 2.4, we will show that, in Theorem 3.1, the Krulltype condition can be replaced by the weaker condition

$$
\operatorname{Int}(D)_{P}=\operatorname{Int}\left(D_{P}\right), \quad \text { for each } P \in t-\operatorname{Spec}(D)
$$

(this is always verified when $D$ is Krull-type by [26, Proposition 2.3]).

We recall several facts that we will freely use in the following.

Remark 3.2. Let $D$ be an integral domain.

(i) If $S$ is a multiplicative subset of $D$, then each contraction to $D$ of a $t$-ideal of $D_{S}$ is a $t$-ideal of $D$ [20, Lemma 3.17].

(ii) Let $Y$ be a nonempty collection of prime ideals of $D$, and let

$$
D^{\prime}:=\bigcap\left\{D_{\mathfrak{p}}: \mathfrak{p} \in Y\right\} .
$$

By applying [13, Proposition 1.3], it follows that, if $\mathfrak{a}$ is a $t$-ideal of $D^{\prime}$, then $\mathfrak{a} \cap D$ is a $t$-ideal of $D$.

(iii) A prime ideal of $D$ which is minimal over a principal ideal is a $t$-ideal [19, page 31, Corollary 3]. In particular, in polynomial rings, the uppers to zero primes are always $t$-ideals.

Lemma 3.3. Let $V \subseteq W$ be valuation domains having the same quotient field, and suppose that $W$ has finite residue field. Then $V=W$.

Proof. There exists a prime ideal $P$ of $V$ such that $V_{P}=W$. If $M_{W}$ is the maximal ideal of $W$, then $M_{W} \cap V=P$, and so, $V / P \subseteq W / M_{W}$. But $W / M_{W}$ is finite, whence $V / P$ is a field. Then $P$ is maximal in $V$ and $V=V_{P}=W$.

Proposition 3.4. Using the above notation, let $D$ be $a \mathrm{P} v \mathrm{MD}$ and $D_{0}$ a Prüfer domain with finite residue fields. Suppose that $\operatorname{Int}(D)_{P}=$ $\operatorname{Int}\left(D_{P}\right)$, for each $t$-maximal ideal $P$ of $D$. Let $i: D \hookrightarrow D_{0}$ be the 
inclusion map and $i^{\star}: \operatorname{Spec}\left(D_{0}\right) \rightarrow \operatorname{Spec}(D)$ the induced contraction sending $\mathfrak{q} \mapsto \mathfrak{q} \cap D$. Then

$$
i^{\star}\left(\operatorname{Spec}\left(D_{0}\right)\right)=\Lambda_{0} .
$$

In particular, it follows that $\Lambda_{0}$ is closed with respect to the constructible topology.

Proof. Let $\mathfrak{q} \in \operatorname{Spec}\left(D_{0}\right)$, and set $P:=\mathfrak{q} \cap D$. Since $D_{0}$ is a Prüfer domain, $P$ is a $t$-prime ideal of $D$, by Remark 3.2 (ii), (iv). Keeping in mind that $D$ is a $\mathrm{P} v \mathrm{MD}, D_{P}$ is a valuation domain such that $D_{P} \subseteq\left(D_{0}\right)_{\mathfrak{q}}$. Furthermore, by Lemma 3.3, we have $D_{P}=\left(D_{0}\right)_{\mathfrak{q}}$ since, by assumption, the residue field of $\left(D_{0}\right)_{\mathfrak{q}}$ is finite. Thus, we have

$$
\operatorname{Int}(D)_{P}=\operatorname{Int}\left(D_{P}\right)
$$

and

$$
\operatorname{Int}\left(D_{P}\right) \neq D_{P}[X]
$$

[2, Proposition I.3.16]. So $P \in \Lambda_{0}$. This proves that $i^{\star}\left(\operatorname{Spec}\left(D_{0}\right)\right) \subseteq$ $\Lambda_{0}$. The converse inclusion is trivial. The fact that $\Lambda_{0}$ is closed with respect to the constructible topology is now clear, in view of $[\mathbf{1}$, Chapter 3, Exercise 27].

Remark 3.5. The last statement of Proposition 3.4 strictly generalizes [3, Lemma 2.6], in which the same result is shown for domains $D$ such that $\operatorname{Int}(D)$ is a $\mathrm{P} v \mathrm{MD}$.

Lemma 3.6. Using the above notation, suppose that $D$ is a $\mathrm{P} v \mathrm{MD}$ such that $\operatorname{Int}(D)_{P}=\operatorname{Int}\left(D_{P}\right)$, for each $t$-maximal ideal $P$ of $D$, and that $D_{0}$ is almost Dedekind with all finite residue fields. Let

$$
i_{0}: \operatorname{Int}(D) \longrightarrow \operatorname{Int}\left(D_{0}\right)
$$

be the inclusion map and

$$
i_{0}^{\star}: \operatorname{Spec}\left(\operatorname{Int}\left(D_{0}\right)\right) \longrightarrow \operatorname{Spec}(\operatorname{Int}(D))
$$

the induced contraction map sending $Q \mapsto Q \cap \operatorname{Int}(D)$. Then,

$$
\left\{M \cap \operatorname{Int}(D): M \in \operatorname{Spec}\left(\operatorname{Int}\left(D_{P}\right)\right), P \in \Lambda_{0}\right\}=i_{0}^{\star}\left(\operatorname{Spec}\left(\operatorname{Int}\left(D_{0}\right)\right) .\right.
$$


Proof. We observe that, if $P \in \Lambda_{0}$, then $D_{P}=\left(D_{0}\right)_{\mathfrak{q}}$, for some $\mathfrak{q} \in \operatorname{Spec}\left(D_{0}\right)$ (Proposition 3.4). In particular, $D_{P}=\left(D_{0}\right)_{D \backslash P}$. Thus, $D_{P}$ is also a localization of $D_{0}$. Then $\operatorname{Int}\left(D_{P}\right) \supseteq \operatorname{Int}\left(D_{0}\right)$, whence we obtain the inclusion

$\left\{M \cap \operatorname{Int}(D): M \in \operatorname{Spec}\left(\operatorname{Int}\left(D_{P}\right)\right), P \in \Lambda_{0}\right\} \subseteq \iota_{0}^{\star}\left(\operatorname{Spec}\left(\operatorname{Int}\left(D_{0}\right)\right)\right.$.

Conversely, let $Q \in \operatorname{Spec}\left(\operatorname{Int}\left(D_{0}\right)\right)$. Then $Q \cap D \in \Lambda_{0}$. In fact,

$$
P=Q \cap D=\left(Q \cap D_{0}\right) \cap D .
$$

By Proposition 3.4, $P \in \Lambda_{0}$. Thus,

$$
Q=Q \operatorname{Int}\left(D_{0}\right)_{D \backslash P} \cap \operatorname{Int}\left(D_{0}\right) .
$$

It is easy to check that $\operatorname{Int}\left(D_{0}\right)_{D \backslash P}=\operatorname{Int}(D)_{P}$. Since $\operatorname{Int}\left(D_{P}\right)=$ $\operatorname{Int}(D)_{P}$, the thesis follows.

Theorem 3.7. Using the above notation, let $D$ be an integral domain such that

$$
\operatorname{Int}(D)_{P}=\operatorname{Int}\left(D_{P}\right),
$$

for each t-maximal ideal $P$ of $D$. Then the following conditions are equivalent:

(i) $\operatorname{Int}(D)$ is a $\mathrm{P} v \mathrm{MD}$;

(ii) $D$ is a $\mathrm{P} v \mathrm{MD}$ and $\operatorname{Int}\left(D_{0}\right)$ is a Prüfer domain.

Proof.

$(1) \Rightarrow(2)$. It is already known.

$(2) \Rightarrow(1)$. Since $D$ is a $\mathrm{P} v \mathrm{MD}, D_{1}$ and $D_{1}[X]$ are also $\mathrm{P} v \mathrm{MDs}[\mathbf{2 0}$, Corollary 3.9 and Theorem 3.7]. Moreover, $\operatorname{Int}\left(D_{0}\right)$ is Prüfer, whence it is a $\mathrm{P} v \mathrm{MD}$. So

$$
\operatorname{Int}(D)=D_{1}[X] \cap \operatorname{Int}\left(D_{0}\right)
$$

is the intersection of two $\mathrm{P} v \mathrm{MDs}$, and, by Corollary 2.15 , it is sufficient to show that $\operatorname{Int}(D)$ is essential with respect to both $D_{1}[X]$ and $\operatorname{Int}\left(D_{0}\right)$.

Take $Q \in t$-Spec $\left(D_{1}[X]\right)$ such that $Q \cap D \neq(0)$. By Remark 3.2 (ii),

$$
Q \cap \operatorname{Int}(D) \in t-\operatorname{Spec}(\operatorname{Int}(D)),
$$


and, by [26, Proposition 2.1],

$$
\mathfrak{p}:=Q \cap D \in t-\operatorname{Spec}(D)=\Lambda_{0} \cup \Lambda_{1} .
$$

If $\mathfrak{p} \in \Lambda_{0}$, then $\operatorname{Int}(D) \nsubseteq D_{\mathfrak{p}}[X]$. But

$$
\operatorname{Int}(D) \subseteq D_{1}[X] \subseteq D_{\mathfrak{p}}[X],
$$

which is a contradiction. It follows that $\mathfrak{p} \in \Lambda_{1}$. We observe that, for such a $\mathfrak{p},\left(D_{1}\right)_{D \backslash \mathfrak{p}}=D_{\mathfrak{p}}$ since $D_{1} \subseteq D_{\mathfrak{p}}$.

Now

$$
D_{1}[X]_{Q}=\left(D_{1}[X]_{D \backslash \mathfrak{p}}\right)_{Q^{e}}=D_{p}[X]_{Q^{e}}=\left(\operatorname{Int}(D)_{\mathfrak{p}}\right)_{Q^{e}} .
$$

If $Q$ is an upper to 0 ideal (in this case $Q K[X]=f K[X]$ for some irreducible $f \in K[X])$, then

$$
D_{1}[X]_{Q}=K[X]_{f}=\operatorname{Int}(D)_{Q \cap \operatorname{Int}(D)} .
$$

Then $D_{1}[X]$ is essential with respect to $\operatorname{Int}(D)$.

Regarding $\operatorname{Int}\left(D_{0}\right)$, take $M \in \operatorname{Spec}\left(\operatorname{Int}\left(D_{0}\right)\right)$. By Lemma 3.6,

$$
M \cap \operatorname{Int}(D)=M^{\prime} \cap \operatorname{Int}(D),
$$

where $M^{\prime} \in \operatorname{Spec}\left(\operatorname{Int}\left(D_{\mathfrak{p}}\right)\right)$, with $\mathfrak{p} \in \Lambda_{0}$. Then

$\operatorname{Int}\left(D_{0}\right)_{M}=\left(\operatorname{Int}\left(D_{0}\right)_{\mathfrak{p}}\right)_{\operatorname{Int}\left(D_{0}\right) \backslash M}=\left(\operatorname{Int}(D)_{\mathfrak{p}}\right)_{\operatorname{Int}\left(D_{0}\right) \backslash M}=\operatorname{Int}(D)_{M \cap \operatorname{Int}(D)}$.

Thus, $\operatorname{Int}(D)$ is essential also with respect to $\operatorname{Int}\left(D_{0}\right)$, and the thesis follows.

Acknowledgments. The authors would like to thank the referee for his/her careful reading of the paper and his/her comments and suggestions.

\section{REFERENCES}

1. M.F. Atiyah and I.G. Macdonald, Introduction to commutative algebra, Addison-Wesley, Reading, 1969.

2. P.-J. Cahen and J.-L. Chabert, Integer-valued polynomials, Amer. Math. Soc. Surv. Mono. 48, Providence, 1997.

3. P.J. Cahen, A. Loper and F. Tartarone, Integer-valued polynomials and Prüfer v-multiplication domains, J. Algebra 226 (2000), 765-787.

4. J.-L. Chabert, Integer-valued polynomials, Prüfer domains, and localization, Proc. Amer. Math. Soc. 118 (1993), 1061-1073. 
5. R. Engelking, General topology, Heldermann Verlag, Berlin, 1989.

6. C.A. Finocchiaro, Spectral spaces and ultrafilters, Comm. Algebra 42 (2014), $1496-1508$.

7. C.A. Finocchiaro, M. Fontana and K.A. Loper, The constructible topology on spaces of valuation domains, Trans. Amer. Math. Soc. 365 (2013), 6199-6216.

8. M. Fontana, Topologically defined classes of commutative rings, Ann. Mat. Pura Appl. 123 (1980), 331-355.

9. M. Fontana and S. Gabelli, On the class group and the local class group of a pullback, J. Algebra 181 (1996), 803-835.

10. M. Fontana, E. Houston and T. Lucas, Toward a classification of prime ideals in Prüfer domains, Forum Math. 22 (2010), 741-766.

11. M. Fontana and A. Loper, The patch topology and the ultrafilter topology on the prime spectrum of a commutative ring, Comm. Algebra 36 (2008), 2917-2922.

12. Nagata rings, Kronecker function rings and related semistar operations, Comm. Algebra 31 (2003), 4775-4805.

13. S. Gabelli, On the Nagata's theorem for the class group, II, Lect. Notes Pure Appl. Math. 206 Dekker, New York, 1999.

14. R. Gilmer, Multiplicative ideal theory, Marcel Dekker, New York, 1972.

15. M. Griffin, Some results on v-multiplication rings, Canad. J. Math. 19 (1967), 710-722.

16. _ Rings of Krull type, J. reine angew. Math. 229 (1968), 1-27.

17. W. Heinzer and J. Ohm, An essential ring which is not a v-multiplication ring, Canad. J. Math. 25 (1973), 856-861.

18. , Noetherian intersections of integral domains, Trans. Amer. Math. Soc. 167 (1972), 291-308.

19. P. Jaffard, Les Système d'Idéaux, Dunod, Paris, 1960.

20. B. Kang, Prüfer v-multiplication domains and the ring $R[X]_{N_{v}}$, J. Algebra 123 (1989), 151-170.

21. A. Loper, A classification of all $D$ such that $\operatorname{Int}(D)$ is a Prüfer domain, Proc. Amer. Math. Soc. 126 (1998), 657-660.

22. A. Loper and F. Tartarone, A classification of the integrally closed rings of polynomials containing $\mathbb{Z}[X]$, J. Commutative Algebra 1 (2009), 51-91.

23. J. Mott and M. Zafrullah, On Prüfer v-multiplication domains, Manuscr. Math. 35 (1981), 1-26.

24. B. Olberding, Globalizing local properties of Prüfer domains, J. Algebra 205 (1998), 480-504.

25. Noetherian spaces of integrally closed rings with an application to intersections of valuation rings, Comm. Algebra 38 (2010), 3318-3332.

26. F. Tartarone, Integer-valued polynomials over Krull-type domains and Prüfer v-multiplication domains, Proc. Amer. Math. Soc. 128 (2000), 1617-1625.

27. M. Zafrullah, On finite conductor domains, Manuscr. Math. 24 (1978), 191204. 
28. M. Zafrullah, The $D+X D_{S}[X]$ construction from GCD-domains, J. Pure Appl. Alg. 50 (1988), 93-107.

Dipartimento di Matematica, Università degli studi Roma Tre, Largo San Leonardo Murialdo 1, 00146 Roma, Italy

Email address: carmelo@mat.uniroma3.it

Dipartimento di Matematica, Università degli studi Roma Tre, Largo San Leonardo Murialdo 1, 00146 Roma, Italy

Email address: tfrance@mat.uniroma3.it 